Voix et Images

volxetimages

\title{
Rhétorique de l'histoire : essai de modèle
}

\section{Pierre Hébert}

Volume 4, numéro 3, avril 1979

Louis-Philippe Hébert

URI : https://id.erudit.org/iderudit/200173ar

DOI : https://doi.org/10.7202/200173ar

Aller au sommaire du numéro

\section{Éditeur(s)}

Les Presses de l'Université du Québec

\section{ISSN}

0318-9201 (imprimé)

1705-933X (numérique)

Découvrir la revue

\section{Citer cet article}

Hébert, P. (1979). Rhétorique de l'histoire : essai de modèle. Voix et Images, 4(3), 492-505. https://doi.org/10.7202/200173ar d'utilisation que vous pouvez consulter en ligne.

https://apropos.erudit.org/fr/usagers/politique-dutilisation/ 


\section{Rhétorique de l'histoire : essai de modèle}

En écho à Valéry, nous reconnaissons de plus è plus que "la littérature est, et ne peut pas être autre chose qu'une sorte d'extension et d'application de certaines propriétés du langage", si bien que toute vision de l'art comme "système modélisant secondaire", c'est-à-dire construit sur le type du langage', fait apparaître l'importance d'examiner, dans le cas des études littéraires, les mutations de la parole en texte.

Notre objet d'étude, dans cet article, sera ce type de discours particulier nommé "discours narratif », qui «ne peut être tel qu'en tant qu'il raconte une histoire, faute de quoi il ne serait pas narratif [...], et en tant qu'il est proféré par quelqu'un, faute de quoi [...] il ne serait pas en lui-même un discours ${ }^{2}$. Nous nous proposons de démontrer qu'une histoire, telle que prise en charge par un discours, reçoit un traitement particulier dont l'analyse s'impose: ce traitement particulier de l'histoire, nous le nommerons rhétorique, désignant par là certaines manifestations du discours narratif, c'est-à-dire le médiateur d'une diégèse. Suite à cette position inaugurale, nous entrevoyons plusieurs questions: parler de rhétorique de l'histoire, n'est-ce pas aussi parler de ses figures? Et, au surplus, comment évoquer les figures sans les accompagner de l'ange déchu, la norme?

Ce sont ces questions que nous allons envisager et auxquelles nous espérons apporter quelques réponses. Nous organiserons cette réflexion autour de trois pôles consécutifs, certes, mais aussi conséquents : l'analyse de l'histoire (et son découpage), l'étude de la temporalité et, enfin, la rhétorique généralisée. Nous présenterons un modèle de découpage du discours narratif, puis nous verrons en quoi ce découpage peut - et doit être - temporalisé et, finalement, le découpage temporalisé nous permettra d'accéder à une taxinomie ${ }^{3}$ des figures de l'histoire. Ces réflexions se grouperont autour d'une première partie, “Propositions». Dans la seconde partie, "lllustrations", nous donnerons quelques exemples de "figures de l'histoire". 


\section{Propositions}

\section{I.1 Pour un découpage du discours narratif}

Les techniques d'analyse de l'histoire, en tant que celle-ci puisse être abstraite du discours, se sont raffinées à un point tel, aujourd'hui, que le catalogue des processus envahit à lui seul une dizaine de pages de Logique du récit de Claude Bremond ${ }^{4}$. Mais si l'établissement des “lois de l'univers raconté " dévoile les principes d'engendrement d'une histoire, il rend compte avec difficulté de la description d'une histoire-occurrence, c'est-àdire de sa prise en charge par un discours.

Pour étayer cette dernière affirmation, suivons Abraham Moles et louri Lotman. Moles distingue deux niveaux de messages, sémantique et esthétique. Le niveau sémantique vise l'intelligible, l'universel; le niveau esthétique désigne l'identifiable ${ }^{5}$. Cette distinction recouvre celle faite par Lotman entre langage et message: "Le langage d'une œuvre donnée [...] existe avant l'élaboration du texte concret [...]. Le message est l'information qui surgit dans un texte donné ${ }^{6}$." Ces coïncidences permettent I'homologie suivante:

$$
\begin{aligned}
\text { niveau sémantique } & =\frac{\text { langage }}{\text { niveau esthétique }} \\
\text { message } &
\end{aligned}
$$

Ainsi Propp et Bremond ont-ils mis au point des modèles dévoilant l'organisation logique du niveau sémantique ou du langage du récit. On sait pourtant que ce langage, l'histoire, n'est toujours donné qu'à travers le discours; ce truisme entraîne une double question : comment, d'abord, découper le discours narratif, c'est-à-dire repérer en texte ses séquences et ses fonctions, et, ensuite, comment établir un inventaire des différentes modalités de la mise en texte (en discours) d'une histoire? Ces interrogations nous invitent à franchir le Rubicon, et à passer du niveau sémantique de l'histoire au niveau esthétique. Aux possibles du langage historique, n'existerait-il pas un équivalent, les possibles du message historique? $\mathrm{Si}$ nous nommons figures certains possibles du message historique qui font écart ${ }^{7}$ à ce qui eût été une présentation "simple et commune " (Fontanier), la question se pose maintenant ainsi : comment découper un récit-occurrence sur le plan de l'histoire et, cela fait, comment établir ses figures?

La première question que nous devrons affronter est donc celle du découpage du discours narratif. II s'agit là d'une.des questions les plus importantes dans l'analyse du récit littéraire. Un texte, quelle que soit sa longueur, se présente toujours comme une totalité massive, sans prise, et force le critique à le diviser, à le fragmenter, pour le saisir avec facilité, ordre et méthode: Genette parle d'une violence inévitable, parce que le discours critique, linéaire lui aussi, ne peut tout dire à la fois. Cette question inaugurale du découpage accompagne forcément celle du contenu diégétique du discours : c'est à partir de moments marqués dans la diégèse qu'on pourra baliser le discours lui-même. Le découpage du discours 
se présente en réalité comme un découpage de l'histoire, et vice-versa. Notre intention n'est pas pour l'instant de tenter un inventaire ou une synthèse de toutes les approches en vue de la segmentation de la diégèse et, par voie de conséquence, dé leur utilisation pour un découpage du discours. Ce labyrinthe nous obligerait à partir de Joseph Bédier, pour passer ensuite à Propp, à Lévi-Strauss, Todorov, Barthes, Greimas et enfin Bremond. Puisque notre but est surtout un découpage fonctionnel du discours narratif, nous proposerons une méthodologie relativement simple qui réclame un court rappel: jetons un regard sur les travaux de Vladimir Propp et de Claude Bremond.

Propp a formulé le vœu, que toue étude historique du conte soit précédée de l'étude des parties constitutives de ce genre littéraire; on pourra alors comparer le sujet des contes en isolant et en confrontant leurs parties constitutives. Propp a certes été le premier à signaler d'une manière aussi claire qu'on peut isoler, des contes, les actions, les fonctions des personnages: dans ces fonctions et dans leur répétition se fondent les éléments permanents du conte: “Par fonction, nous entendons l'action d'un personnage, définie du point de vue de sa signification dans le déroulement de l'intrigue ${ }^{8}$."

Claude Bremond, généralisant la démarche de son prédécesseur russe, recherche les lois ou les contraintes logiques de l'univers raconté dans un récit. L'atome narratif est alors la fonction: une séquence résulte du groupement de trois fonctions précises, une qui ouvre (conduite à tenir, événement à prévoir), une qui réalise (conduite, événement en acte) et une dernière qui clôt (résultat atteint). Les deux dernières fonctions peuvent ne pas se réaliser, ou réaliser leur. contraire (exemple : résultat non atteint). Enfin, les séquences peuvent s'enchaîner soit bout à bout, soit par enclave, soit par accolement.

Notre tâche prend maintenant forme : il s'agit pour nous de découper le récit en séquences élémentaires en repérant séquences et fonctions dans le texte. Il faut entendre.par séquence élémentaire tout groupement des trois fonctions amélioration possible/processus (ou non)/amélioration obtenue (ou non) ou encore du type dégradation possible/processus (ou non)/dégradation obtenue (ou non). Mais pensons à aller plus loin que le découpage de l'histoire : celui-ci'doit scinder le discours lui-même. Les réalisations des séquences et des fonctions en discours découperont le récit et nous donneront un découpage premier ${ }^{9}$ du récit..

Il s'agit donc de faire passer un modèle conçu pour le découpage de l'histoire au niveau du discours narratif. L'entreprise est à plusieurs égards périlleuse' (il est souvent difficile de localiser les séquences et les fonctions en texte) mais elle permet une saisie globale du récit assez intéressante. Propp avait déjà soupçonné un tel investissement de son modèle dans. son dernier chapitre, “Le conte comme totalité »; où il se rapproche du discours. 
On nous dira: mais à quelle «épaisseur» du texte un tel découpage s'exerce-t-il ?. Est-il microscopique? macroscopique?

Pertinente à bien des égards, cette question doit être envisagée avec beaucoup de prudence : comment être assuré que nous sommes en possession du «bon » découpage?

Nous nous sommes reporté à cette unité de découpage que constitue la séquence: celle-ci relate une suite d'événements qui ont un début (projet), un. milieu (processus) et une fin (réalisation) ${ }^{10}$. II s'agit donc d'un projet qui s'achemine logiquement. Or, nous proposons de repérer la séquence au niveau de l'interstructure ${ }^{11}$, lieu médian entre la macro et la microstructure. La macrostructure désigne c̀es grandes articulations narratives que, par exemple. Genette isole dans la Recherche de Proust: à peine' onze pour toute l'œuvre ${ }^{12}$. La microstructure a aussi tróuvé une illustration chez Genette, au début du chapitre de l'ordre ${ }^{13}$. On se contente alors de mettre en relief les plus petites unités narratives. L'interstructure a céci de particulier qué, se distinguant par le sens du récit, elle s'intègre à la macrostructure, de même qu'elle intègre la microstructure, comme le monème intègre le phonème tout en s'intégrant lui-même à la phrase.

Le découpage, unique et absolu, n'existe pas: l'important est de disposer d'un découpage ayant une valeur opératoire, d'un découpage qui ne soit pas nécessairement vrai,' mais juste.

\section{I.2 Pour une temporalité du découpage du discours narratif}

Les réflexions que' nous venons de faire sur l'histoire libèrent maintenant une interrogation sur le temps. Les études sérieuses sur le sujet sont légion: il suffit de rappeler les travaux de Pouillon, Poulet, Weinrich, Genette, entre autres. Toutefois, ces approches de la temporalité semblent souvent en quête d'objet: le temps est toujours temps de quelque chose, dans le récit comme ailleurs, mais de quoi ? Une solution possible se trouve chez Bremond (dont le modèle nous suivra tout au long dé cet article), dont les propos 'invitent à établir une synergie' fonctionnelle entre les études de I'histoire et celle du temps, deux études qui se sont contentées de se saluer d'un rivage à l'autre:

L'éventualité, le passage à-l'acte, le résultat d'un processus sont évidemment relatives à un système de repères chronologiques : à tel instant de l'histoire racontée, que nous nommerons $t$, tel processus est présenté comme achevé (il s'est développé dans un temps $t-n$ ), tel autre est en cours (son actualisation coïncide avec t), tel autre est dans les limbes (il passera à l'acte, peut-être, à l'instant $t+n)^{14}$.

Cette remarque réclame la liaison entre histoire et temporalité, fạisant de celle-ci l'objet de celle-là. Et cette connexion ne sera possible, bien sûr, que si l'histoire est repérée en discours : hors du discours, point de temps. Ainsi, puisque l'histoire d'un récit littéraire est connaissable grâce au modèle de Bremond, même dans l'usage élémentaire que nous en proposons, 
il faut maintenant chercher à savoir pourquoi nous pouvons temporaliser les séquences et les fonctions. Nous venons de voir que Bremond soupçonnait la dimension temporelle intrinsèque de son modèle: au sujet de la séquence, il parle d'une série «temporelle structurée", en opposition à la chronologie, quand les événements sont intégrés dans un projet humain. Mais cette temporalité dépasse celle de l'histoire (bien qu'elle s'y inscrive) et rejoint celle du discours : nous lisons souvent les études sur le temps du récit en les transposant inéluctablement (et souvent inconsciemment) dans les termes de Bremond. Ainsi Jean-Jacques Mayoux : «Chaque microcosme a sa temporalité close et séparée, présentant sa structure propre qui règle son rythme et son flux ${ }^{15}$. " On perçoit l'importance, après avoir repéré ces microcosmes, c'est-à-dire les séquences et les fonctions, d'établir leur temporalité. Mayoux poursuit: “l'écrivain créateur, qui est un créateur de temps et non seulement d'une histoire en termes logiques, a mis son temps en mouvement $[\cdots]^{16}$. "Lisons aussi Robbe-Grillet avec les mêmes yeux :

Les passions comme les événements ne pouvaient être envisagés [dans le roman traditionnel] que dans un développement temporel: naissance, croissance, paroxisme, déclin et chute. Tandis que dans le récit moderne, on dirait que le temps se trouve coupé de sa temporalité. II ne coule plus. II n'accomplit plus rien ${ }^{17}$.

C'est hors de tout doute la temporalité des séquences et des fonctions qui peut permettre de vérifier les dires de Robbe-Grillet. Claude Bremond, dans son article célèbre, écrivait : “L'étude sémiologique du récit peut être divisée en deux secteurs : d'une part, l'analyse des techniques de narration, d'autre part, la recherche des lois qui régissent l'univers raconté ${ }^{18}$. "Bremond a choisi, c'est connu, l'étude des lois de l'univers du récit et de l'inventaire des possibles narratifs. Or nous avons proposé, à partir de son modèle, de déborder l'histoire pour envahir le discours, c'est-à-dire non seulement de tenter un repérage des séquences en discours, mais surtout de les temporaliser, ce qui revient à étudier les relations d'ordre, de durée et de fréquence qui peuvent marquer les fonctions et les séquences. C'est sa temporalité qui peut donner au récit ses critères distinctifs. Que Rodrigue ne se fût pas complu dans ses stances, et nous aurions eu la même logique : problème à résoudre, processus, problème résolu. Mais c'est dans la mesure où la fonction "problème à résoudre" occupe une durée privilégiée que l'histoire trouve sa temporalité spécifique: temps de l'attente, de l'angoisse, diront certains critiques. Ce sont précisément ces possibles de spécification temporelle que nous ambitionnons de décrire.

Nous venons de proposer des relations de mesure d'ordre, de durée et de fréquence. Justifions maintenant ce choix.

Il semble que l'on puisse, pour préciser les relations de mesure, dégager deux types d'êtres temporels: ceux qui durent dans le temps audelà du temps même de leur apparition, et ceux qui ne durent que le temps minimal nécessaire à leur perception. On pourrait parler, dans ce premier 
cas, d'une temporalité non discrète et dans le deuxième, d'une temporalité discrète. On sait que l'on entend par unité discrète, en linguistique par exemple, une unité qui n'admet pas la gradation. Le signe linguistique peut donc être dit "discret" (nous reprenons Martinet), tandis que, par exemple, les couleurs d'une carte topographique sont des unités non discrètes : telle couleur, plus ou moins foncée, indique un changement de relief. Cette distinction se révèle indispensable pour l'analyse d'une séquence typographique régulière. Alors, dans la catégorie du non discret (du temps extensible) on pourrait citer en exemple une séquence diégétique : ainsi le triptyque narratif amélioration possible/processus d'amélioration/amélioration obtenue, dans le discours narratif, se présente incarné dans le rapport discours narratif/histoire, et peut au surplus durer un certain temps. Deux relations temporelles sont alors pertinentes: d'ordre et de durée. La seconde catégorie, celle de la quantité discrète, peut être illustrée par les signifiants d'un personnage. Ainsi le signifiant “Élisabeth», dans Kamouraska, ne peut avoir qu'une durée invariable ${ }^{19}$ : une seule relation temporelle apparaît pertinente: l'ordre d'apparition de ce signifiant dans le discours narratif. Cependant - et cette remarque ajoutera une nouvelle relation temporelle - les deux types de temporalité décrits plus haut, discrète et non discrète, sont susceptibles de se répéter dans le discours narratif. Rien ne nous garantit qu'une même fonction (au sens de Bremond), qu'un même signifiant ne sera pas réitéré à un moment ou l'autre dans le discours. Dès lors, une nouvelle dimension temporelle se présente sous le scalpel de l'analyste: la fréquence. Selon la nature de l'objet étudié, trois relations temporelles sont donc possibles: ordre, durée et fréquence. Ces réflexions pourraient être regroupées dans le tableau suivant:

\begin{tabular}{|c|c|c|c|}
\hline \multicolumn{2}{|r|}{ Type d'objet } & Exemple & Relation temporelle \\
\hline 1. & $\begin{array}{l}\text { Temporalité non } \\
\text { discrète }\end{array}$ & Séquence, fonction & Ordre, durée \\
\hline 2. & Temporalité discrète & $\begin{array}{l}\text { Signifiant d'un personnage, } \\
\text { intrusion du narrateur }\end{array}$ & Ordre \\
\hline & Catégories 1 et 2 & - & Fréquence \\
\hline
\end{tabular}

On comprend maintenant la nécessité des notions d'ordre, de durée et de fréquence. Ces trois procédés de mesure désignent les relations élémentaires temporelles. L'étude des séquences et des fonctions dans un discours suppose une étude de l'avant et de l'après, parce que "l'information contenue dans un message est déterminée par le pouvoir qu'il a de s'organiser selon un ordre particulier ${ }^{20}$ ", réduisant du même coup son entropie. La durée se pose également comme norme de mesure parce que "la progression accélérée de certains thèmes impose forcément aux autres le retard [...]. Le temps seul peut fondre en chœur les chants isolés ${ }^{21}$ ». Enfin, la fréquence elle-même ne peut être négligée, non seulement parce que l'on connaît l'importance de la périodicité dans la perception, non seulement également parce que la complexité détermine le degré d'in- 
formation d'un texte, mais aussi - et surtout parce qu'il s'agit de saisir, par la fréquence, la répartition des figures d'un texte afin de déterminer «si le lot [...] se localise au hasard dans l'étendue du texte ou non $[\ldots]^{22}$. “La fréquence d'un fait de langue ne devrait jamais être séparée de la stabilité de cette fréquence, de la répartition plus ou moins régulièrè du fait 23, .

\section{I.3 Pour les figures de l'histoire}

Le lien que nous venons d'établir entre le découpage et la temporalité nous sert à présent d'assise pour l'établissement des figures de l'histoire. Mais la figure étant ce qui s'écarte de "l'expression simple et commune", la question qui se pose maintenant est celle de la norme comme critère de différenciation.

La pluralité des normes, qui évince “l'existence d'une norme, d'un idéal général et absolu, et l'historicisme, incompatible avec le panchronisme, auraient causé la mort de la rhétorique, et plus particulièrement du couple figure-norme, selon Todorov 24 ". Sa mort? Non, sa retraite temporaire. La résurgence de la rhétorique se fait aujourd'hui à la faveur du rétablissement de la norme quantitative ${ }^{25}$, ou, plus simplement, de son enracinement «en dehors du langage ${ }^{26}$ ", de même que par une conversion du "panchronisme" classique en "poétique" moderne, c'est-à-dire la mise sur pied du discours littéraire comme principe d'engendrement des textes.

Ressusciter la norme, pour établir les figures de !'histoire, exige que nous cueillions les contours de celle-ci hors du contenu diégétique luimême. Nous éviterons donc de nous appuyer, par exemple, sur le vraisemblable de l'histoire, nous rejetterons également les modalités de la mimésis (représentation) et de la diegesis (récit pur) sélectionnés par une époque donnée. Et c'est dans la temporalité que nous arc-bouterons la norme.

Dès lors, il s'ensuit que l'histoire ou mieux, ses séquences et ses fonctions, peuvent manifester un triple écart : d'ordre, de durée et de fréquence, si bien que les normes elles-mêmes seront :

- d'ordre: les séquences présentent-elles un ordre rigoureux des fonctions ouverture - processus - résultat? trop peu?

- de durée: chacune des trois fonctions pèche-t-elle en trop ou en

- de fréquence: I'une ou l'autre de ces trois fonctions est-elle réitérée?

Que le récit se déroule sans atteinte à l'ordre, à la durée et à la fréquence des fonctions, et il ne présentera alors aucune figure (ce qui est d'ailleurs à peu près improbable...). 
Sans doute a-t-on perçu l'importance de la temporalité comme charnière entre les fonctions-occurrences et les figures de l'histoire. Récusant alors l'expression "déformation temporelle", nous parlerons plutôt de "formation temporelle», si bien que c'est sur cette formation que reposent les palpitations du récit:

L'analyse actuelle tend en effet à “déchronologiser» le contenu narratif et à le «relogifier » [...]. On pourrait dire d'une autre façon que la temporalité n'est qu'une classe structurelle du récit [du discours], tout comme dans la langue, le temps n'existe que sous forme de système; du point de vue du récit, ce que nous appelons le temps n'existe pas, ou du moins n'existe que fonctionnellement, comme élément d'un système sémiotique ${ }^{27}$.

Nous tentons précisément, dans cette recherche, de nous évader du temps référentiel, et d'accéder à une conception renouvelée du temps, fondée sur la mesure.

Faut-il craindre les taxinomies? Non, si elles sont un instrument de connaissance. Le rapport entre les fonctions-occurrences et la temporalité fait apparaître les figures pọssibles de l'histoire, que nous résumons dans le tableau suivant :

Les possibles des figures de l'histoire

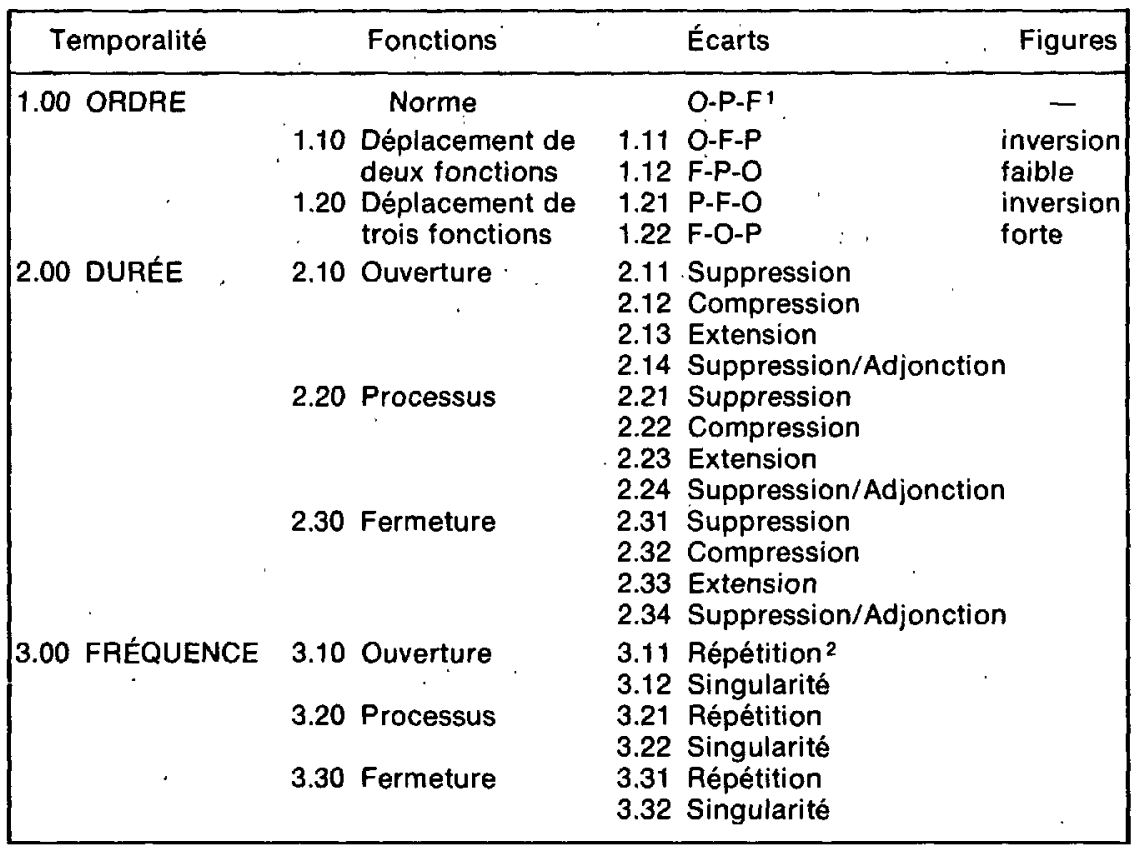

1. $\mathrm{O}, \mathrm{P}$ et $\mathbf{F}$ signifient "fonctions ouverture, processus et fermeture" ".

2. Nous souscrivons aux définitions de Genette concernant les définitions de récits singulatifs et itératifs (Figures III). 
Nous ne sommes pas le premier à proposer une rhétorique de l'histoire : le groupe $\mu^{28}$ et Maurice-Jean Lefebvre ${ }^{29}$ l'ont fait avant nous.

L'effort du groupe $\mu$ était d'ailleurs très englobant: il portait sur presque toutes les instances du récit. Cependant, en ce qui a trait aux figures de l'histoire, la norme apparaissait plus ou moins claire et, au surplus, les "relations de durée" et les «faits de la chronologie empiétaient les uns sur les autres. D'autre part, ce qui gêne dans l'entreprise de Lefebvre, c'est l'absence d'une norme rigoureuse : le degré zéro «aura même un aspect quelque peu mythique. Impossible, en effet, de le définir autrement que comme la "réalité quotidienne», acceptée et reçue par nous, à une époque donnée et dans une situation donnée, comme normale, habituelle, incontestée $[. . .]^{30}$ ».

C'est de là que nous avons ressenti la nécessité de fonder la norme sur la temporalité : l'écart devient à ce moment mesurable et, ainsi, tend à s'évader de l'historique.

La prochaine tâche qui nous attend est d'émailler cette rhétorique par divers exemples. En suivant la taxinomie proposée, nous examinerons au moins un cas de figure par fonction. Nous choisirons des exemples opportuns pour l'illustration, sans égard à la série culturelle à laquelle ils appartiennent, puisque en définitive, c'est l'exemplarité qui prévaut.

\section{Illustrations}

Afin de ne pas trop alourdir cette section, nous choisirons seulement quelques exemples de figures. Nous croyons, en effet, qu'une figure de fonction par norme temporelle suffira à illustrer les propositions que nous avons avancées et à démontrer leur valeur heuristique. Ainsi pouvons-nous proposer les exemples suivants :

1.00 ORDRE :

1.10 Déplacement de deux fonctions, inversion faible (P-O-F) : “L'Héritage", de Ringuet.

1.20 Déplacement de trois fonctions, inversion forte (P-F-O) :

1.21 Poussière sur la ville, d'André Langevin.

2.00 DURÉE :

2.13 Extension de la fonction ouverture :

Madame Bovary, de Gustave Flaubert.

2.24 Suppression/Adjonction de la fonction processus : "Le Chat noir», d'Edgar Poe.

2.32 Compression de la fonction fermeture :

"Le Chat noir", l'Étranger (Camus), Au-delà des visages (Giroux). 


\subsection{FRÉQUENCE :}

3.11 Répétition, fonction ouverture :

Poussière sur la ville.

3.22 Singularité, fonction processus : La Peste, d'Albert Camus.

3.31 Répétition, fonction fermeture :

Poussière sur la ville.

\subsection{ORDRE}

\subsection{0 “L'Héritage», de Ringuet}

Ringuet a écrit un recueil de contes, l'Heritage ${ }^{31}$, qu'un critique a d'ailleurs rapproché du Torrent d'Anne Hébert. Or, le conte inaugural de ce recueil, "l'Héritage", présente une séquence (inaugurale elle aussi) du type P-O-F. En effet, le récit nous fait voir Albert arrivant à la campagne, à la recherche de la terre que lui a léguée en héritage son père Baptiste. Ce n'est pas du pourquoi dont nous sommes d'abord informés, mais du quoi: la fonction ouverture, qui nous entraînerait du côté des causes de cette arrivée, ne surgira qu'après l'arrivée elle-même. Ce type de mise en situation ne va donc pas sans conséquences importantes: l'intérêt narratif se trouve d'emblée projeté vers le déroulement même de l'action, au détriment des ressorts premiers de l'histoire.

\subsection{Poussière sur la ville, d'André Langevin}

On retrouve, dans ce récit ${ }^{32}$ de Langevin, un cas frappant d'inversion forte (P-F-O), au début de la deuxième partie, inversion qui présente le déroulement suivant :

\footnotetext{
$P$ (de dégradation): Mouvement de révolte d'Alain (p. 95).

$\downarrow$

F (dégradation obtenue) : "Noir devant, noir derrière, noir partout" (p. 96).

$\downarrow$

O (dégradation possible) : Alain aperçoit Madeleine (p. 96).
}

Cette configuration, en plus de mettre en exergue l'importance de la "crise", des conséquences, retarde de deux temps la cause même de cette réaction. Double effet, donc: relief donné au comportement, et intrigue nouée autour de la cause de ce processus et de ce résultat.

\subsection{DURÉE}

\subsection{Madame Bovary, de Gustave Flaubert}

L'extension de la fonction ouverture désigne le possible temporel le plus intéressant. Elle correspond, grosso modo, à une excroissance du "que faire?", inventorié en rhétorique sous les noms de délibération, opta- 
tion, suspension, etc. II faudra pourtant distinguer ces deux cas : soit que “l'auteur s'attarde à décrire des faits insignifiants et à multiplier les notations chronologiques ${ }^{33}$ ", soit que la fonction soit allongée sans le recours de notations non fonctionnelles : nous touchons alors à ce que Barthes appelle "catalyse", qui "accélère, retarde, relance le discours, le résume, anticipe, parfois même déroute ${ }^{34}$ ". Madame Bovary propose un bel exemple: Emma, dès la page 31, commence à rêver, à espérer du nouveau (amélioration possible, donc ouverture) ; cet état - quand même dynamique - dure jusqu'à la page $43^{35}$. Cette excroissance est essentielle au récit : elle y instaure une prédominance du souhaité sur le vécu, exploité sous le terme «bovarysme". Cette figure fondamentale, Jean-Pierre Duquette n'a pas manqué de la relever dans son étude de l'Éducation sentimentale ${ }^{36}$ : "temps du temps qui passe", "temps de l'éventuel", expressions qui montrent bien que l'excroissance de la fonction ouvrante opère un effet destructeur chez les personnages flaubertiens.

\subsection{4 «Le Chat noir », d'Edgar Poe}

Le récit peut présenter un type particulier de digression où ala relation narrateur/lecteur au détriment de la relation narrateur/récit ne peut qu'apparaître comme détournement du temps ${ }^{37}$ ". Cette ctechnique du languissement" consiste à remplacer le temps historique par -un temps subjectif. A ce titre les commentaires du narrateur dans son récit où il est autodiégétique, lors d'un processus de malfaisance dans le Chat noir d'Edgar Poe, paralysent le temps objectif: "Je tirai de la poche de mon gilet un canif, je l'ouvris; je saisis la pauvre bête par la gorge, et, délibérément, je fis sauter un de ses yeux de son orbite. Je rougis! Je brûle, je frissonne en écrivant cette damnable atrocité ${ }^{38}$ ! Cet arrêt pourrait se manifester au cinéma par une fixation de l'image sur le processus malfaisant, accompagné de la parole du narrateur : la durée s'abolit dans l'intemporel.

\subsection{2 . "Le Chat noir ", l'Étranger, Au-delà des visages}

II n'est pas difficile de trouver des exemples de compression de la fonction « résultat " : dans "le Chat noir", il faut deviner l'arrestation du narrateur; dans l'Étranger, il faut imaginer l'exécution de Meursault, tout comme dans Au-delà des visages, d'André Giroux. Cette interruption du processus témoigne à sa manière du «sujet» du récit, qui ne doit pas être cherché dans le résultat: aussi l'Étranger n'est-il pas un récit sur la peine capitale, et le roman d'André Giroux nous oblige-t-il à polariser notre lecture du côté des intérêts narratifs bien avant ceux de l'histoire.

\subsection{1 et 3.31 Poussière sur la ville}

Nous conjuguons ici deux types: la répétition des fonctions d'ouverture et de fermeture. C'est que l'exemple choisi, le début de Poussière 
sur la ville, présente des séquences qui réalisent ces deux cas. Voici l'ouverture du récit :

1. DP = Avertissement de Kouri (p. 11).

$P \quad=$ Alain devient angoissé (p. 11).

$\overline{\mathrm{DO}}=$ Fuite : " Je quittai le restaurant sans mot dire " (p. 12).

2. $D P=$ Alain repense à l'avertissement (p. 12).

$P \quad=$ Alain soupçonne Kouri d'avoir "levé le rideau " (p. 13).

$\overline{\mathrm{DO}}=$ Fuite : "Je n'ai ni le goût, ni le temps de ces sortes de jeux" (p. 13).

3. $D P=$ "Qu'a voulu dire le Syrien?" (p. 13).

$P \quad=$ Alain songe à Madeleine, souvent seule chez Kouri (p. 13).

$\overline{D O}=$ "Cela ne concerne que Madeleine et moi » (p. 13).

Nous épargnons au lecteur une énumération des autres séquences. Remarquons cependant les fonctions d'ouverture et de fermeture: les seize premières séquences du récit, jusqu'à la page 85 , sont constituées de onze fonctions d'ouverture et de fermeture identiques:

Ouverture : avertissements (de Jim, trois, et de Kouri, huit).

Fermeture: mouvements de fuite d'Alain: "Cela ne concerne que Madeleine et moi » (p. 13); “le Syrien est assez simple d'esprit» (p. 14); «je ne songe guère à cela" ( $p .19$ ); etc.

Convenons qu'il s'agit là d'une forme originale de répétition, de réitération d'un même événement. Le récit, en son début, présente un fait déterminant, l'avertissement, qui fait que la narration fouille, reprend, retourne, avec une allure piétinante.

\subsection{La Peste}

Pour clore cette partie des exemples, nous proposons d'observer, dans la Peste ${ }^{39}$ de Camus, la singularité de la fonction de processus.

Les premières séquences dè la Peste sont toutes constituées par l'apparition des rats, qui entraîne un processus de dégradation. Ces processus, considérés dans leur identité, se présentent ainsi :

P. 1 : «La présence de ce rat mort lui avait paru seulement bizarre tandis que, pour le concierge, elle constituait un scandale» (p. 9).

$P$. 2: "Intrigué [par la présence de rats], Rieux décida de commencer sa tournée $[\ldots] »$ (p. 10).

P. 3: L'apparition des rats "finit par être agaçante», dit Rieux (p. 13).

P. 4: “Ces rats lui avaient donné [au concierge] un dur coup [...]" (p. 14).

Le récit continue ainsi : ouverture par l'apparition de rats, processus de dégradation, clôture par des mouvements de négation. Poussière sur la ville nous avait présenté la narration plurielle d'un événement unique; ici, nous avons la narration plurielle d'événements pluriels identiques. 


\section{Conclusion}

Nous sommes convenus, dans ce court article de considérer le récit littéraire comme un discours narratif où le narratif, le diégétique, reçoit un traitement particulier par le discours. Ce traitement, nous l'avons appelé “rhétorique». Nous croyons que le modèle d'une rhétorique de l'histoire que nous venons de proposer possède suffisamment de généralité pour rendre compte des possibles narratifs sur le plan discursif. Nous n'oserions prétendre que tous les possibles s'y rencontrent; mais nous croyons que le modèle et, plus particulièrement, les principes qui l'inspirent (norme et temporalité) sous-tendent l'organisation discursive de l'univers narratif.

Pierre Hébert, Université de Sherbrooke.

1. Voir à ce sujet louri Lotman, la Structure du texte artistique, Paris, Gallimard NRF, 1973, p. 52.

2. Gérard Genette, Figures III, Paris, Seuil, 1972, p. 74.

3. "ll arrivera un jour où la rhétorique ne sera plus qu'une énumération de figures" (T. Todorov, Theories du symbo/e, Paris, Seuil, 1977, p. 65). Rejetant les conceptions pragmatiques, ornementales et affectives de la rhétorique, nous voulons plutôt dresser un inventaire des possibles narratifs discursifs.

4. Claude Bremond, Logique du récit, Paris, Seuil, 1973, p. 335-344.

5. Abraham Moles, Art et ordinateur, Paris, Casterman, 1971, p. 35.

6. Iouri Lotman, op. cit., p. 44.

7. Voilà un gros mot, et qui appelle une définition que nous donnerons plus loin.

8. Vladimir Propp, Morphologie du conte, Paris, Seuil, “Points", 1970, p. 31.

9. La division du texte en séquences diégétiques est appelée ici première, non pas parce qu'elle apparaît de soi, car il est bien évident que le recours à un modèle d'analyse interdit une telle affirmation, mais bien parce que la fable est essentielle au récit. Qu'on se souvienne d'Aristote ("Les actes et la fable sont la fin de la tragédie; et c'est la fin qui en toutes choses est le principal m) et, 2000 ans plus tard, d'un formaliste russe, Tomachevski (“Le héros n'est guère nécessaire à la fable. La fable comme système de motifs peut entièrement se passer du héros et de ses traits caractéristiques").

10. On se rappellera que le processus et la réalisation peuvent ne pas avoir lieu.

11. Nous empruntons cette idée à M. N.-Lachapelle, mémoire de M. A., Sherbrooke, 1974.

12. Gérard Genette, op. cit., p. 124-125.

13. Ibid., p. 83 et suiv.

14. Claude Bremond, op. cit., p. 311-312.

15. Jean-Jacques Mayoux, "Temps vécu et temps créé dans Tristam Shandy", dans Poétique, $n^{\circ} 2$, p. 175.

16. Ibid., p. 180.

17. Alain Robbe-Grillet, Pour un nouveau roman, Paris, Gallimard, 1972, p. 168.

18. Claude Bremond, "la Logique des possibles narratifs", dans Communications, $n^{\circ} 8,1966$, p. 60.

19. Étant bien entendu par là, cependant, que la durée signifie le nombre de lignes ou, analogiquement, de lettres dans un mot. Ce qui occupe un espace $x$ prend un temps $x$ ' à l'occuper.

20. Umberto Eco, l'CEuvre ouverte, Paris, Seuil, 1965, p. 76. 
21. Nelly Cormeau, Physiologie du roman, Paris, Nizet, 1966, p. 106.

22. Charles Muller, Initiation à la statistique lexicale, Paris, Larousse, 1968, p. 43.

23. Ibid., p. 18.

24. Tzvetan Todorov, op. cit., p. 138.

25. Pensons à Jean Cohen, Structure du langage poétique.

26. Groupe $\mu$, Rhétorique générale, Paris, Larousse, 1970, p. 37.

27. Roland Barthes, "Introduction à l'analyse structurale des récits", dans Communications, $n^{\circ} 8,1966$, p. 12.

28. Groupe $\mu$, op. cit.

29. Maurice-Jean Lefebvre, Structure du discours de la poésie et du récit, Neuchatel, éd. de la Baconnière, 1971.

30. Id., “Rhétorique du récit ", p. 128.

31. Ringuet, l'Héritage, Montréal, éd. Variétés, 1946.

32. André Langevin, Poussière sur la ville, Montréal, Cercle du livre de France, 1953.

33. Groupe $\mu$, op. cit., p. 182.

34. Roland Barthes, op. cit., p. 10.

35. Gustave Flaubert, Madame Bovary, Paris, Garnier-Flammarion, 1966.

36. Jean-Pierre Duquette, Flaubert ou l'architecture du vide, Montréal, Les Presses de l'Université de Montréal, 1972.

37. Groupe $\mu$, op. cit., p. 179.

38. Edgar Allan Poe, ale Chat noir », Paris, Garnier, 1961.

39. Albert Camus, la Peste, Paris, L.G.F., "Livre de poche ", 1968. 